\title{
Importance of Crop Altitude Range for Coffee Production: Findings from Honduras
}

\author{
Carmen Peligros-Espada ${ }^{1}$, Joel Ulises Sevilla-Palma ${ }^{2}$ and Octavio Uña-Juarez ${ }^{1}$ \\ 1. Rey Juan Carlos University, Camino del Molino, s/n, Fuenlabrada 28942, Madrid, Spain \\ 2. SAG EmprendeSur, Colonia Loma Linda, Avda. La FAO, Boulevard Centroamerica, Tegucigalpa 11101, Honduras
}

\begin{abstract}
The aim of this paper was to show the empirical relationship found between the coffee crop altitude and the attributes of the coffee. Data from Honduras were used, but findings can be extended to global coffee production. In this way, the production of speciality coffee identified locally with a good potential at final markets can be promoted. From the launch of the National Coffee Policy in Honduras, one special interest was given to improve the speciality coffee' national production, and the Honduran Institute of Coffee (IHCAFE) turned into a key actor to assume the leadership in the execution of the policy, particularly in the local assistance and the international promotion of the coffee. From gathering a national coffee quality project, IHCAFE collected hundreds of samples out of the producing regions of the country from four consecutive annual harvest (2004-2008), the followed quality analysis found diverse profiles of flavors and aromas of the coffee, and also a relevant link between quality features and geographical, agronomic and technical determinants (flavor, aromas, altitude range, agronomic variety and producing region).
\end{abstract}

Key words: Agriculture, coffee attributes, altitude, Honduras.

\section{Introduction}

During the 19th century, the coffee was promoted as a commodity [1-3] and was increasingly requested by a growing population at final markets after the end of Second World War-when the trade was re-established-so the Honduran authorities encourage production and exports [4-8].

Motivated by markets, producing countries promote reforms to better support coffee production [9-12]. In Honduras, the international demand transformed the coffee from an uncommon crop to a major mainstream business at the end of the 20th century $[9,10,13]$.

Ignited by a new cycle of high volumes and low prices at international markets, a worldwide coffee crisis was developed from 2000 to 2004 [14-17], so the Honduran Institute of Coffee (IHCAFE) developed strategic, economic and technical actions to promote production and increase the national revenue obtaining better prices from differentiated niche coffees. Among

Corresponding author: Carmen Peligros-Espada, Ph.D., research fields: agriculture, geography and economy. these actions, IHCAFE led the development of the National Quality Center, improving coffee analysis before export, promoting coffee competitions, such as Cup of Excellence, and celebrating marketing campaigns at final markets, such as Specialty Coffee Association of America (SCAA) [18, 19]. The market price for specialty coffee is generally higher than the regular commodity price, even if the coffee come from the same country. So, the origin does not seem to determine quality, but rather the specific features of the coffee. The coffee has a segmentation based on quality for the two main species: Arabica and Robusta. According to the International Coffee Organization (ICO), there are four main types of coffee available in international market: Colombian mild Arabica, other mild Arabica, Brazilian and other natural Arabica and Robusta [10, 18, 20].

Honduras was responsible in 2015 [17] for nearly $4 \%$ of the total worldwide production of 8.7 million tons of green coffee. A nation of only $112,492 \mathrm{~km}^{2}$ is the seventh out of the top ten worldwide coffee producers. In contrast, Honduras fits the profile of the 
United Nations of a developing country, reflecting a mid-low human development index, all together with social problems, poverty and heavy dependence on light manufacturing, agriculture and raw material exports [21]. According to the Honduran Central Bank, coffee was the main export commodity in 2015 with 986 million USD, representing $26 \%$ of total exports freight on board (FOB) and roughly 5\% of GDP [17, 22]. Internally, while primary production kept increasing, the quality control was problematic after the harvest and particularly during the wet milling processing of fruits to parchment, leaving coffee parchment without a proper drying and with high humidity content, besides promoting the presence of fungus, provoking a fast deterioration of coffee quality during transport, storage and trade. With a complicated scenario, different studies had found related quality problems within the Honduran coffee industry, among them: insufficient technology transference, poor processing facilities and above all complicated marketing relationships between growers and intermediaries [13, 23].

The general objective of this research was to highlight the relationships among some Honduran coffee characteristics and some technical and geographical features (flavor, fragrance, altitude range, agronomic variety and coffee region), finding the links among these variables and, in this way, promote Honduran coffee quality. So, the relationship between flavor and fragrance is going to be discovered and the relationship among coffee altitude range, agronomic variety, producing region and coffee characteristic (body, flavor, acidity and aftertaste) is going to be explored.

In order to achieve this goal, several determinants of coffee quality from the consumers' point of view have been taken into account.

\section{Materials and Methods}

\subsection{Study Area}

Honduras provides excellent climatic and agricultural conditions for coffee production, mountain ranges covering most of the interior land of Honduras, crossing the country from east to west, facilitating coffee production in several geographical locations [23]. The production of coffee is well distributed within the mountain ranges of Honduras [23], allowing several producing regions with potential for specialty or differentiated coffee: Montecillos, Azul Meambar, Opalaca, Copan and Agalta Tropical, as appeared in Fig. 1. These regions have been selected following IHCAFE's nationwide distribution, which includes the location of field technicians and facilities to process and to store coffee samples at the time. Within the Honduran producing regions, 173 coffee samples originated from Agalta Tropical, 203 from Azul Meambar, 208 from Copan, 184 from Montecillos and 136 from Opalaca. Samples came from three altitude ranges: low/less than 900 masl), medium (901-1,200 masl) and high (1,201 masl and above).

\subsection{Sample Size Estimation}

The coffee quality had been tested by cuppers prior to the commercial contract settlement in the international market [8], but it has been especially after the international coffee crisis and the rising of specialty coffees, the market improves coffee evaluation methods and industry starts to standardize cupping procedures to differentiate coffee [17, 24]. During this time, coffee quality became a motivating factor for consumers and business, and for a more scientific approach involving the physical evaluation of the coffee infusion [17, 24, 25]. Some empirical works were related to analyze coffee quality to origin [20]; others had linked quality to the chemistry of coffee [26, 27], or even to do evaluation of the cupping protocols [28].

The sample size estimation was based on a universe of 67,868 growers and followed by a well-known statistical protocol with expected error of 0.015 and confidence of 90\% [29, 30]. From 2004 to 2008, a 


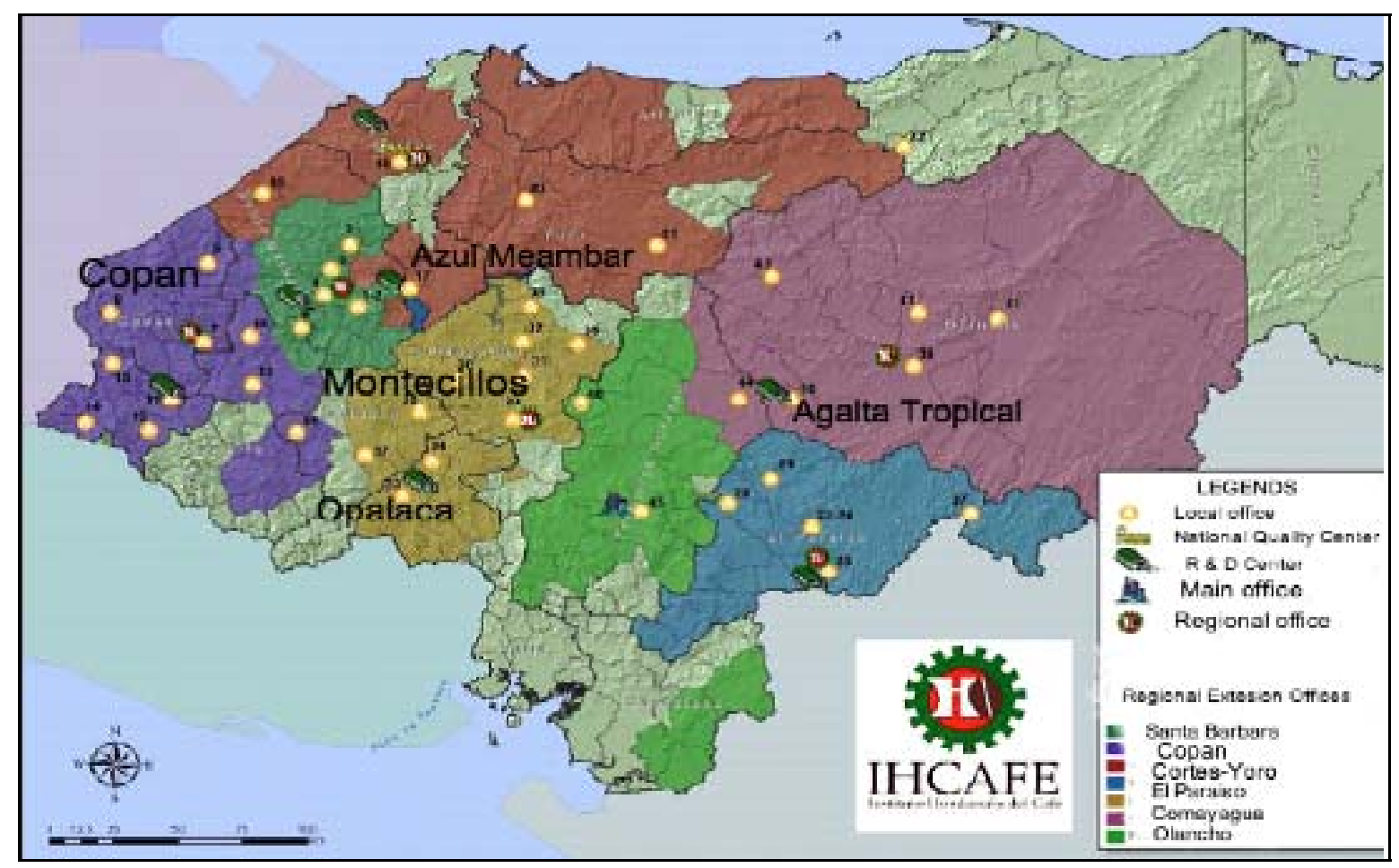

Fig. 1 Coffee regions and national distribution of IHCAFE.

High altitude range: Opalaca and Montecillos; Medium: Azul Meambar; Low: Copan and Agalta Tropical.

Source: IHCAFE.

total of 904 coffee samples were approved for research purpose of IHCAFE analysis [10, 23].

\subsection{Cupping Method}

All samples were out of arabica coffee varieties-Bourbon, Catuai, Caturra, Colombia, Geisha, IHCAFE-90, Lempira, Pacas, Typica and Villa Sarchi-and were collected in dry parchment during the harvest season (from October to April of the following year), transported directly from farmers, centralized and stored in sealed plastic bags. Samples in parchment were prepared within a month, before obtaining a medium roast product by the quality control lab (IHCAFE). The samples were ground medium a few minutes before obtaining the infusion used in the sensory test room for cupping evaluation. The amount of ground coffee used for the infusion was set up to obtain an optimum ratio of $8.25 \mathrm{~g}$ of coffee per $150 \mathrm{~mL}$ of water. Ground coffees were put in white ceramic cups, and hot purified water was poured over the ground coffee $\left(93^{\circ} \mathrm{C}\right.$, reverse osmosis and carbon filtered water), some 3-5 min before cupping by panelist from IHCAFE's quality control lab. The sensory test followed IHCAFE criteria, based on SCAA protocol recommendations.

The coffee samples were roasted a day before they were ground, allowing the coffee to rest at less $8 \mathrm{~h}$ before cupping by panelists. The water used for cupping was odor free, neither distilled nor softened, within the ideal total dissolve solids; neither less than 100 ppm nor more than 250 ppm. The fresh water was brought to approximately $93{ }^{\circ} \mathrm{C}$ before being poured directly onto the ground coffee up to the rim of the ceramic cups (set of five). The infusion was left undisturbed for 3-5 min before the panelists did the cupping evaluation.

The perception of coffee's quality was the main reason for the sensory test done by cuppers. Once the cups were presented to the cuppers, the procedure included smelling, breaking the crust, re-smelling and testing the coffee flavors (at around $71{ }^{\circ} \mathrm{C}$ ). The attributes of coffee samples were: fragrance, flavor, 
aftertaste, acidity, body, balance, uniformity, clean cup, sweetness, defects and overall. The attributes were rated on a numeric scale within a cupping form. The specific flavor attributes are positive or negative scores of quality, reflecting the judgment of cuppers (defects were unpleasant or negative flavors). It is aware that the quality of the coffee can mean different things to different people, and the perception of the flavor of the coffee is a complex phenomenon which involves olfactory sensations, taste and touch. The coffee quality is defined by the consumer taste, at the end, if the infusion has a pleasant taste to the consumer [31].

\subsection{Statistical Methods}

For this study, by the amount of information and the number of variables used, performing analysis on coffee can be non-metric and non-quantity type of information, since for more traditional ways, statistical analysis can be challenging using qualitative type of information. Then, the related databases on the coffee qualitative information can be analyzed by using multivariate analysis techniques. The statistical program InfoStat ${ }^{\circledR}$ was used for multivariate analysis of the data information, popular software for natural and agricultural science analysis in Latin America [10, 32, 33].

Following the multivariable statistical methods, the correspondence analysis was primarily selected from fundamental multivariable techniques to perform interdependency analysis, in which the initial contingency tables were later used to present a graphical plot visualizing the relationships between selected variables.

The multivariable information generated by the cupping analysis of coffee samples and done by IHCAFE's quality control lab, was analyzed during this study by using the "InfoStat" computer program [10, 32, 33]. Among the variables, some were the quantitative variables, representing the geographical origin of coffee samples "regions" and the quantitative variables evaluated directly by IHCAFE, resulting in prime flavors and fragrances coming from the coffee infusion evaluation. The variables then were evaluated by using correspondence analysis technique [10, 25]; other variables were also collected and used for this study, such as "meters above sea level” (quantitative), "altitude ranges" (qualitative), "precipitation" (quantitative) and "agronomic coffee varieties" (quantitative).

It is necessary in multivariable analysis to separate dependent and independent variables. Specifically, it is necessary when those relate to coffee characteristics and describe the relationship to each other. Commonly in coffee research, this is quite difficult [10, 34]. Following the multivariable statistical methods, commonly being used to analyze quality rather than quantity type of information, the correspondence analysis was primarily selected to perform interdependency analysis, in which the initial contingency tables were later used to present a graphical plot visualizing the relationships between selected variables [32, 35]. Other multivariable analysis techniques were complementary to the study: principal components (PCs) technique to perform simultaneous analysis of quality and quantity variables and the canonical correlation using only quantity types of variables.

To evaluate interdependency among variables, cluster analysis could be used to reduce sample size and to seek similarities or differences within the groups of observations, following the hypothesis under Pearson's chi-square statistic. In correspondence analysis, the plot of points of each row and each column of the contingency table is a projection of two-dimensional Euclidean space, all in order to preserve the distance between rows or columns, if rows or columns are not similar [33].

In correspondence analysis, the case of a column being closer to a row point, the two are less independent if the combination of categories of the two variables occurs more frequently than just by 
chance. So, following the matrix algebra, a contingency table of " $a$ " rows and " $b$ " columns, $n_{i j}$ are the frequencies for the every cell combination [33, 35].

\section{Results and Discussion}

3.1 Coffee Quality: Relationship between Flavor and Fragrance

A reduction of information among variables related to taste has been made due to the initial consideration of too many flavor and fragrance profiles. It was made by selecting the occurrence frequency of $5 \%$ or more within the total observations from the initial 26 categories of prime fragrances in coffee. They were reduced to: chocolate, 317 observations (35\% of the time); sweet, 259 (29\%); citric, 90 (10\%); fruity, 56 (6\%); peanuts, 44 (5\%) and the rest to other fragrances and other defects. In the case of the prime flavors, identification was given according to $5 \%$ or more in occurrences out of the initial 43 categories and these were reduced to: citric, 242 observations (27\%); chocolate, 229 (26\%); sweet, 144 (16\%); fruit (6\%); peanuts (6\%) and the rest to other flavors and other defects.

Following the correspondence analysis, the study exhibited a relationship between some variables using the normal approach of confidence interval with a 95\% coefficient. The analysis found statistical significance between the variables "prime flavor" and "prime fragrance", that is to say the dependence among variables flavor and fragrance is significant. As shown in Table 1, the value of the chi-square distribution was $66.85 \%$, so the null hypothesis of independence was rejected; and the value of the total inertia is $75 \%$, so the flavor can be explained by the fragrance, but also for other variables.

It can be seen that the highest association was chocolate flavor with chocolate fragrance with $18.25 \%$ of the time, $8.63 \%$ of the time sweet flavor relating to sweet fragrance, $1.77 \%$ of the time fruit flavor relating to fruit fragrance, $7.08 \%$ of the time citric fragrance relating to citric flavor and $2.88 \%$ of the time peanut fragrance relating to peanut flavor (both defects). Complementarily, the analysis showed that $9.07 \%$ of the time sweet flavor was related to citric fragrance and $0.66 \%$ of the time chocolate fragrance was related to citric flavor, sweet flavor was related to chocolate fragrance $5.53 \%$ of the time, fruity flavor was related to citric fragrance $2.21 \%$ of the time. The other relationships found were $2 \%$ or less of the relative frequency between variables. Moreover, of the 144 values of the contingency table, 59 are null values, that is to say, in the $41 \%$ of cases, there is no relationship between flavor and fragrance.

The negative flavors as peanuts, grassy, astringent were in relationship to the damaging practice of wet parchment coffee trading and the internal trading of coffee, dominated by the middlemen. The processing of coffee without the appropriate drying has been problematic, particularly during the wet milling of cherries to parchment, leaving coffee parchment with high humidity content, besides promoting the presence of fungus, provoking a rapid deterioration of quality during most of the transport, storage and trade [11-13, 36].

It is necessary to improve coffee technology transference and processing facilities to get a better regulation of marketing relationship between growers and intermediaries, particularly poor and complicated $[10,13,36,37]$. There is a damaging practice of wet parchment coffee trading between producers and dominating middlemen. The processing of coffee by the wet method has been problematic, particularly during the wet milling of cherries to parchment, leaving coffee parchment with high humidity content, without the appropriate drying, besides promoting the presence of fungus, provoking a rapid deterioration of quality during most of the transport, storage and trade of coffee. Without sufficient processing, quality control and technical assistance, the formation of moulds causes defects affecting the inner quality of coffee [11-13]. 


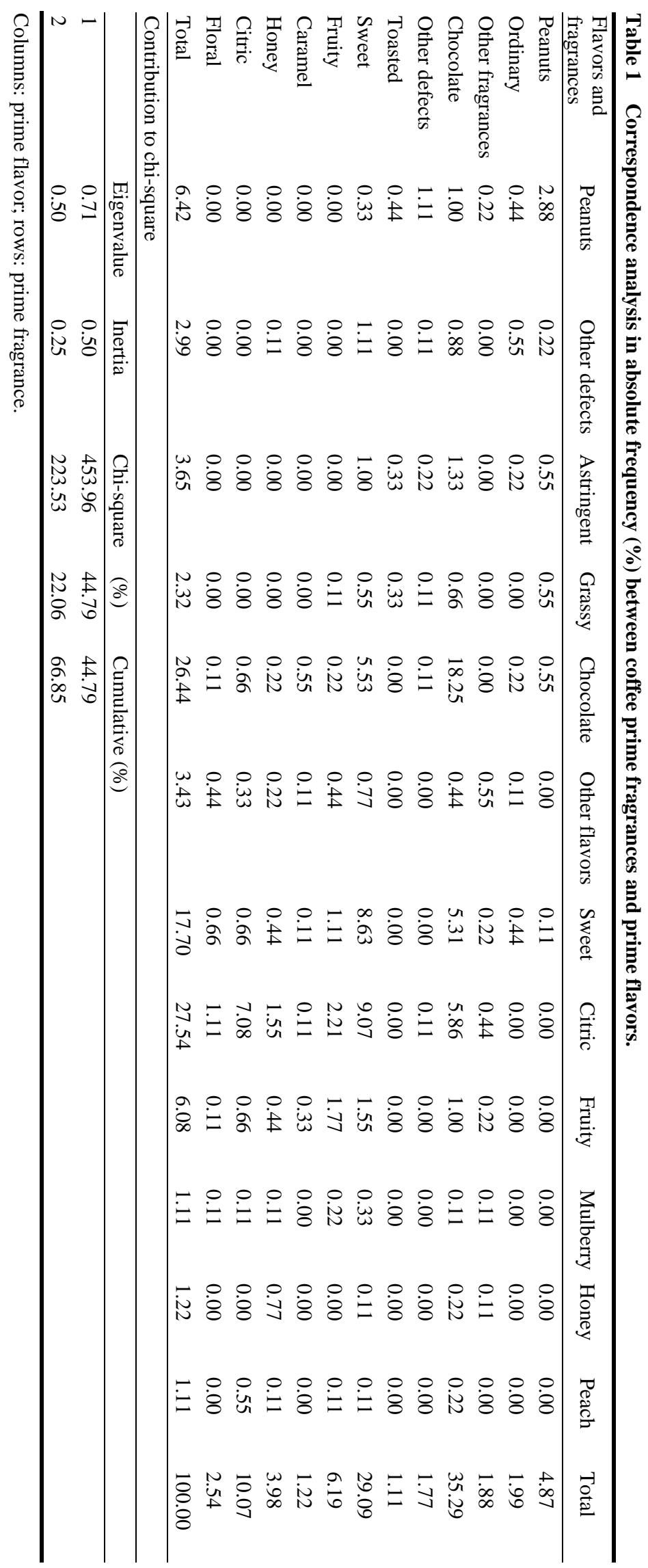


In the case of the quality program fulfilled by IHCAFE, policy actions aiming at preserving coffee quality were found, besides the export quality control, such as the effort to achieve the International Standards Organization (ISO), accreditation of technical competence for coffee quality analysis and the international promotion of differentiated coffees [10, 23, 38, 39]. However, no actions have been registered to address direct quality improvements on Honduran mainstream coffee within the present internal trade of wet parchment coffee [10, 36]. A better processing and more quality control could have differentiated prices.

Following a national trend for the pursuit of the specialty coffee market, this study found supporting evidence of the diverse and positive profiles on the flavors and aromas coming from the Honduran coffee. These coffee's flavors and aromas were found to be linked to crop altitude ranges, opening new possibilities to address investments and technical assistance to growers.

3.2 Relationship between Coffee Altitude Range, Agronomic Variety, Producing Regions, Fragrances and Flavors

In Fig. 2, from the correspondence analysis technique with inertia of $78.89 \%$ of chi-square distribution, the analysis found a statistical relationship between variable "altitude range" in horizontal axis (red circle) and variable "coffee agronomic variety": "low" altitude range was associated to the Pacas variety; "high" altitude range was associated to Caturra and Catuai varieties; "medium" altitude range was associated to Lempira and IHCAFE-90 varieties and to Villa Sarchi variety.

In Fig. 3, with an inertia of $84.06 \%$ of the chi-square distribution in the horizontal axis 1 , the correspondence analysis found a statistic relationship between the variable "regions" in columns (blue square) and variable "altitude range" in horizontal axis (red circle): "medium" altitude range was better related to coffee producing regions of Copan; the "high" altitude range was associated with the regions of Opalaca and Montecillos, both in the central part of Honduras; the "low" altitude range was associated to Agalta Tropical in the eastern part of the country. A perfect match was found between correspondence analysis and geographical data.

In Fig. 4, with a chi-square distribution of $71.66 \%$ of inertia in the horizontal axis 1, the analysis found a statistical relationship between variable "prime fragrance" (blue square) and variable coffee crop

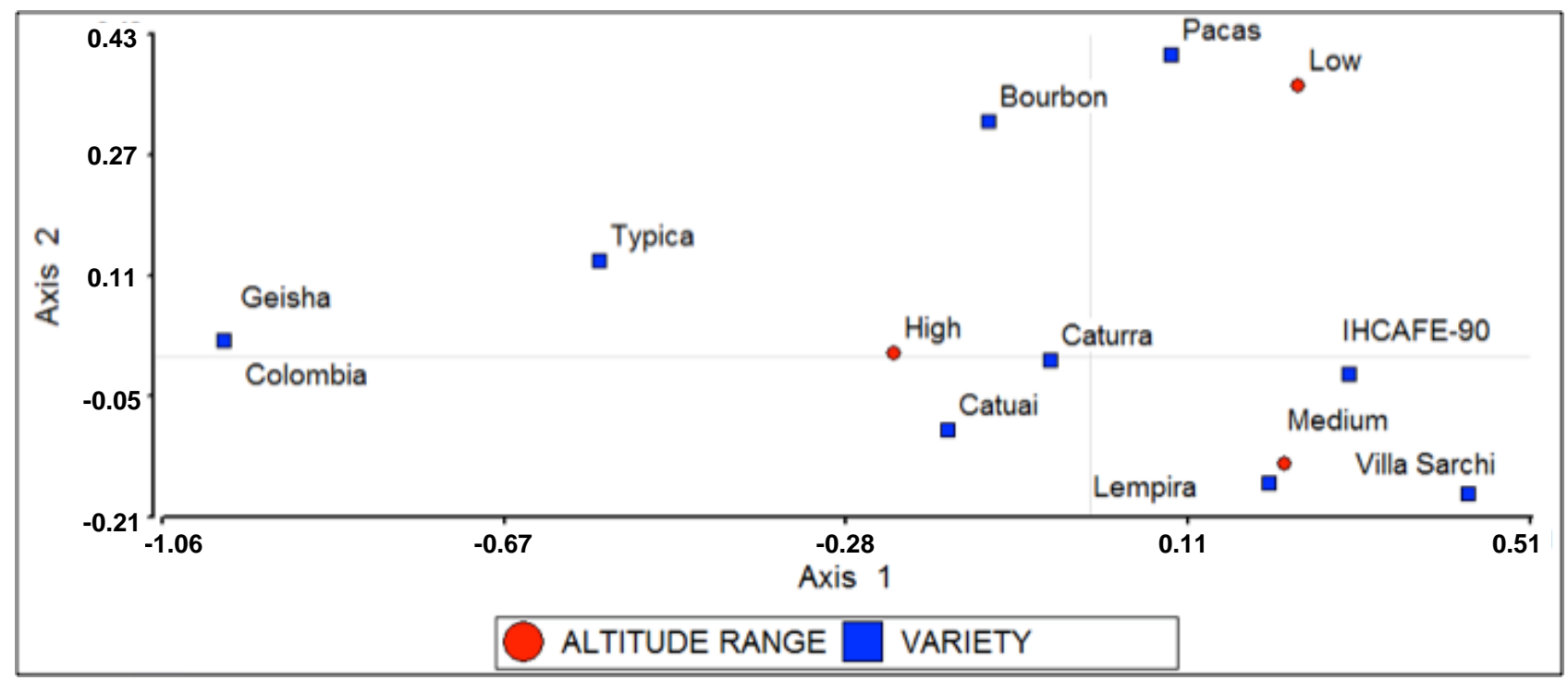

Fig. 2 Correspondence analysis between coffee crop altitude ranges and agronomic varieties. 


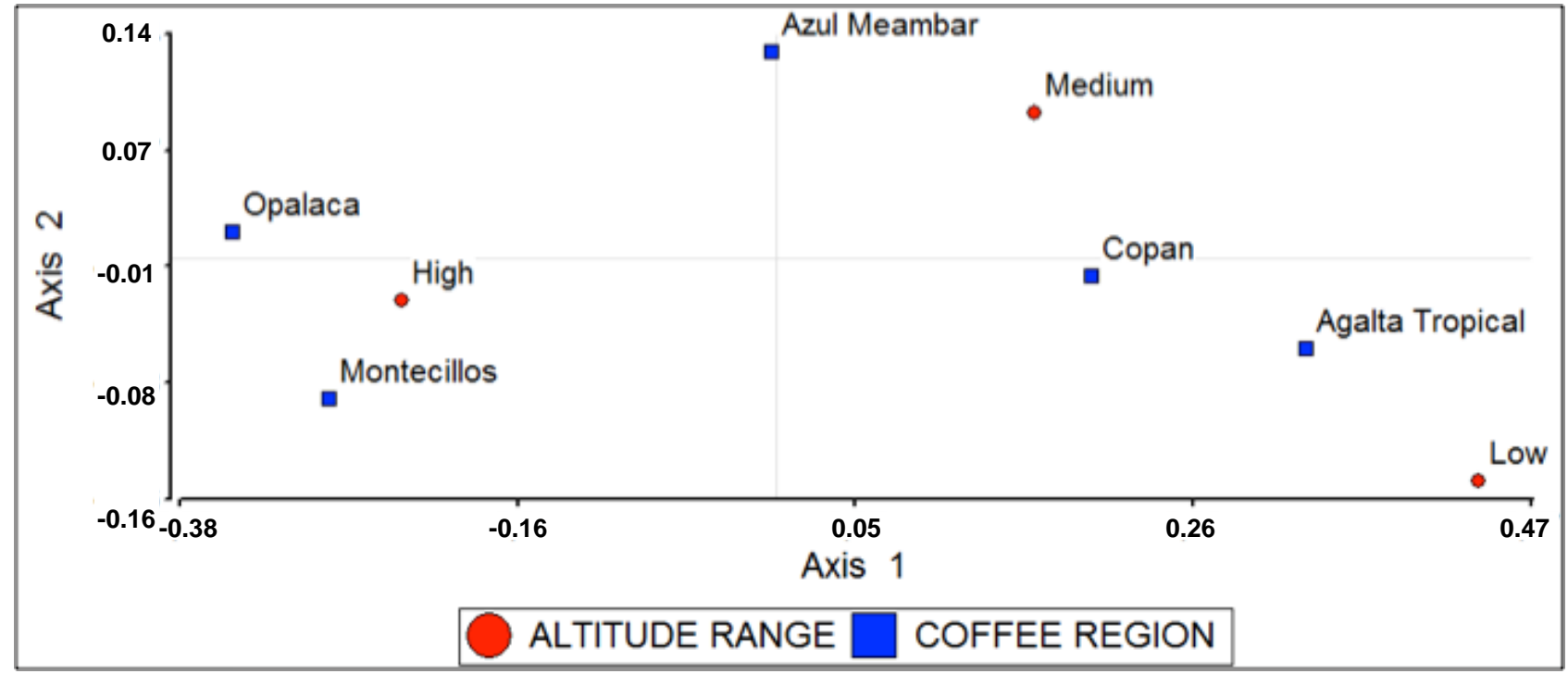

Fig. 3 Correspondence analysis between altitude ranges and coffee producing regions.

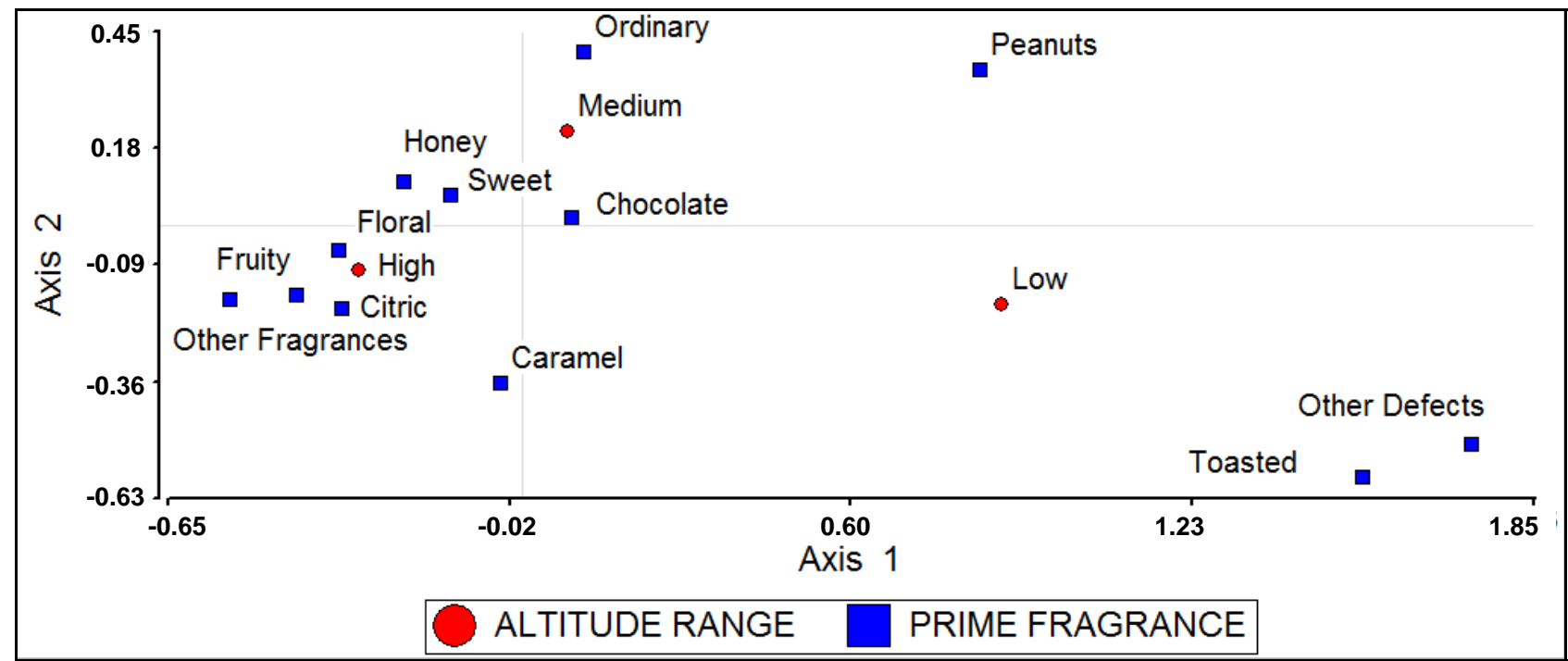

Fig. 4 Correspondence analysis between coffee altitude ranges and coffee prime fragrances.

“altitude range” (red circle): “medium” altitude range was more closely associated with chocolate and ordinary fragrances; "high" altitude range was more closely associated with citric, floral, fruity and other fragrances (known positive attributes); "low" altitude range was distant from peanuts.

In Fig. 5, with a chi-square distribution of $94.94 \%$ in the horizontal axis 1 , the analysis found statistical significance between variable "altitude range" in horizontal axis (red circle) and variable "flavor" in columns (blue square): "medium” altitude range was associated with chocolate, grassy and sweet flavors; “high” altitude range was closely associated to citric, fruity, honey, peach and other flavors; finally, "low" altitude range was associated with peanuts flavor.

3.3 Relationship between Coffee Altitude Range and Coffee Characteristic: PCs and Canonical Correlation

In Fig. 6, PCs analysis presented the horizontal component "PC" with $82.2 \%$ and the vertical component with 15.5\%, in total explaining $97.7 \%$ of the model variability; whereas, the coffee characteristics: aroma, body, flavor, acidity and 


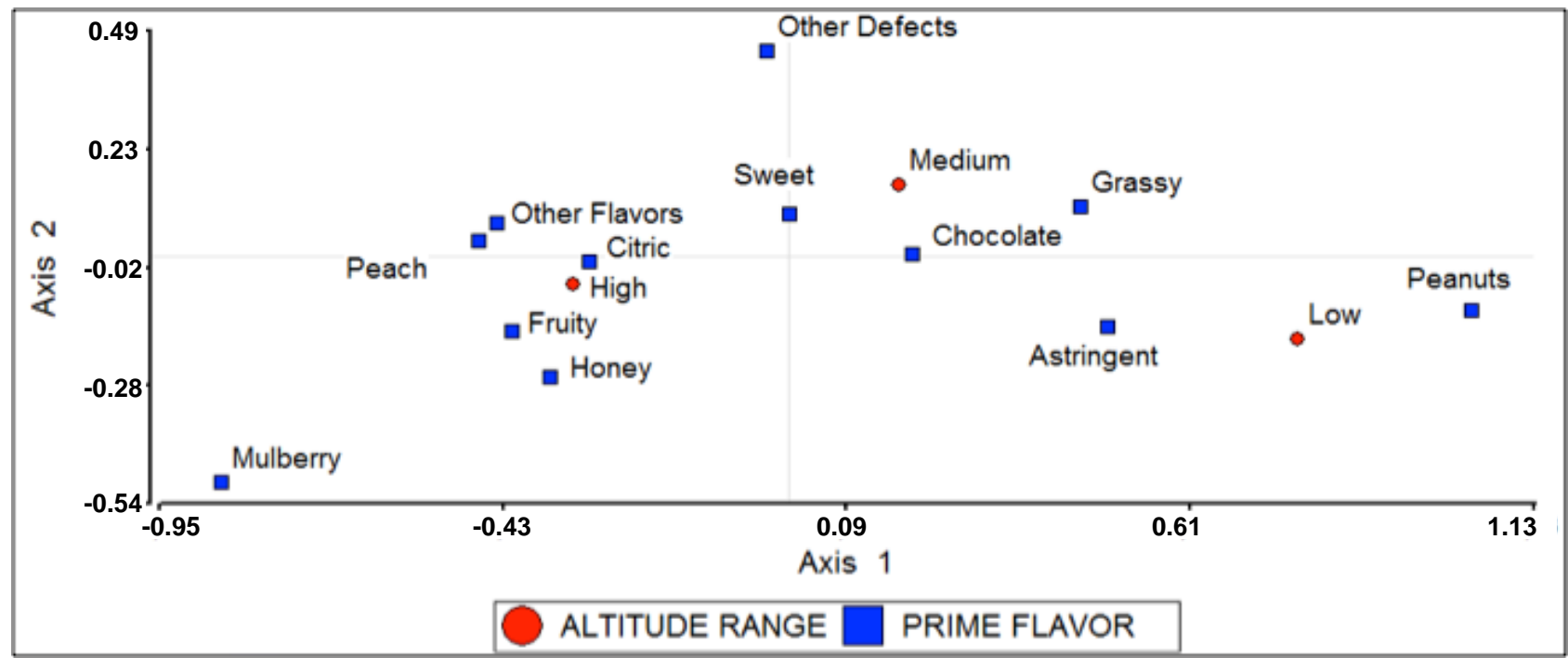

Fig. 5 Correspondence analysis between coffee altitude ranges and coffee flavors.

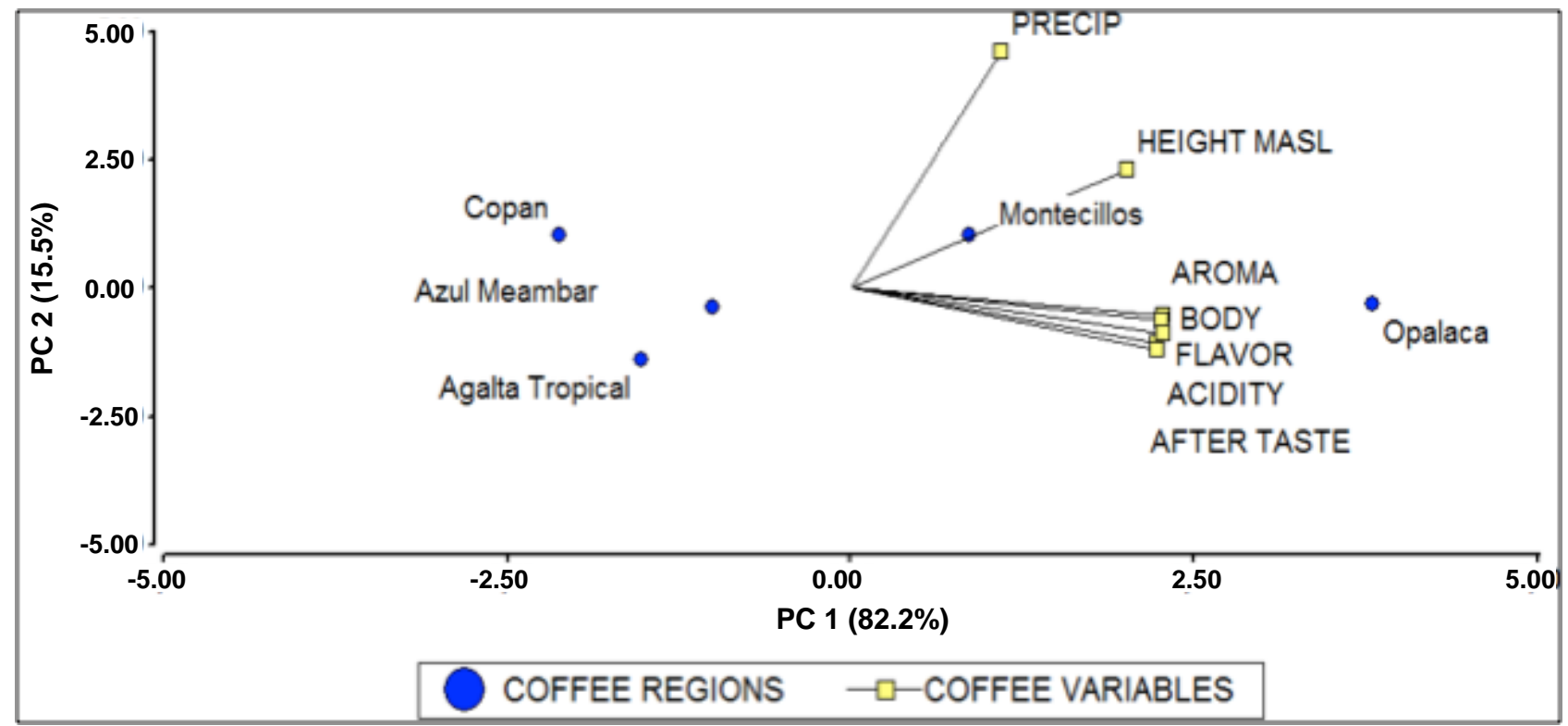

Fig. 6 PCs analysis between coffee regions and coffee characteristics.

aftertaste were closely related to each other, the variables coffee crop height in meters above sea level "height masl” and rain precipitation in mm "precip" were distant and independent. The coffee growing region of Montecillos was related to coffee crop height and Montecillos along with Opalaca were both associated with the coffee characteristics.

In the covariance matrix of Table 2, the importance of the height can be seen, so the variable "height masl" is positively correlated with aroma, body, acidity, flavor and aftertaste, and negatively correlated with rainfall. Acidity is the most appreciated characteristic in the commercialization in coffee and with better commercial value [31]. Also, empirical evidences of a negative relation were found between rain precipitation and height. Also, note how the magnitude of the covariance of the variable "height masl” with every other variables.

In Table 2, the multivariate analysis presented by the first canonical correlation $\mathrm{L}(1) R$ was 0.52 of the first pair of canonical correlations, and $R \quad 0.27$ represented a variability of $27 \%$ of the data explained 
Table 2 Canonical correlation between environmental variables and coffee characteristics.

\begin{tabular}{|c|c|c|c|c|c|c|c|}
\hline Covariance matrix & Height masl & Precip & Aroma & Body & Acidity & Flavor & Aftertaste \\
\hline Height masl & $58,083.50$ & $-1,770.40$ & 58.54 & 66.48 & 86.36 & 90.61 & 87.84 \\
\hline Precip & $-1,770.40$ & $36,119.92$ & -3.04 & -4.11 & -7.05 & -5.28 & -5.11 \\
\hline Aroma & 58.54 & -3.04 & 0.28 & 0.25 & 0.32 & 0.34 & 0.34 \\
\hline Body & 66.48 & -4.11 & 0.25 & 0.32 & 0.37 & 0.40 & 0.39 \\
\hline Acidity & 86.36 & -7.05 & 0.32 & 0.37 & 0.49 & 0.50 & 0.49 \\
\hline Flavor & 90.61 & -5.28 & 0.34 & 0.40 & 0.50 & 0.56 & 0.54 \\
\hline Aftertaste & 87.84 & -5.11 & 0.34 & 0.39 & 0.49 & 0.54 & 0.54 \\
\hline \multicolumn{8}{|l|}{ Canonical correlation } \\
\hline & $\mathrm{L}(1)$ & $\mathrm{L}(2)$ & & & & & \\
\hline$R$ & 0.52 & 0.06 & & & & & \\
\hline$R^{2}$ & 0.27 & 0.0034 & & & & & \\
\hline Lambda & 281.22 & 3.09 & & & & & \\
\hline Degrees of freedom & 10.00 & 4.00 & & & & & \\
\hline$p$ value & 0.00 & 0.54 & & & & & \\
\hline
\end{tabular}

by the correlation among quantity variables: coffee crop altitude in meters above sea level "height masl", precipitation in mm "precip" and coffee characteristics: aroma, body, acidity, flavor and aftertaste. The first canonical correlation between scores in quantitative and non-quantitative classes is different from zero in the population with a $p$-value of less than 0.001 .

\section{Conclusions}

The main finding of this study was that the empirical evidence suggested the importance of crop altitude for coffee cultivation. Evidence was found in the relationship between altitude ranges, agronomic varieties, producing regions and coffee fragrances and flavors. Moreover, it was found that coffee attributes (aroma, body, flavor, acidity and aftertaste) were closely related to each other and also related to the "height masl".

The finding is explained fully below in four conclusions:

(1) This study is able to offer supporting evidence of the diverse profiles of flavors and fragrances of the Honduran coffee by the correspondence found between coffee prime fragrances and flavors. Cuppers detected these prime flavors: peanuts, ordinary, other fragrances, chocolate, other defects, toasted, sweet, fruity, caramel, honey, citric and floral; and these prime fragrances: peanuts, other defects, astringent, grassy, chocolate, other flavors, sweet, citric, fruity, mulberry, honey and peach. This is important, especially for differentiated coffees among the Japanese and US markets, where positive attributes of fragrance and flavor are highly appreciated. Furthermore, the rich profile of flavor and fragrance found in coffee produced in Honduras is supportive of any international marketing campaign or better pricing strategies for the international trade.

(2) These coffee flavors and fragrances are in relationship with crops altitude range.

The empirical evidence was found suggesting the importance of crop altitude for coffee cultivation. In the coffee world, particularly in the specialty coffee segment, it is a well-known fact that coffees coming from higher growing altitudes have a tendency to score higher in quality appreciation, flavors and aromas.

The empirical evidence is found between altitude range and coffee flavor. The common link "altitude range” recognizes the importance of the altitude above sea level related to flavors. Low altitude is related to peanuts, while high altitude is related to positive attributes, such as fruity, citric and other flavors. These results are confirmed by the covariance matrix, so the variable "height masl" is positively correlated 
with aroma, body, acidity, flavor and aftertaste variables, and negatively correlated with rainfall. It is found that the coffee at highest altitude has a tendency to receive less amount of rain precipitation.

Empirical evidence was found between altitude range and coffee fragrance. Low altitude was related to peanuts, medium altitude was related to chocolate and ordinary fragrance, and high altitude was related to citric, floral, fruity and other fragrances (known positive attributes)

Also, the empiric evidence suggested the relationship between altitude ranges and flavors: low altitude was related to peanuts flavor; medium altitude was related to grassy, chocolate and sweet; high altitude was related to other flavors (positive attributes), citric, peach and fruity.

The coffee attributes (aroma, body, flavor, acidity and aftertaste) are close related to each other and also related to the height in meters above sea level and rain precipitation. These coffee attributes are also related with the regions of Montecillos and Opalaca, range mountains with the highest altitude.

Finally, in the canonical correlation, a positive relationship was found between the variable height masl and the variables aroma, body, acidity, flavor and aftertaste. A negative relationship was found between height masl and rain precipitations variables, showing that coffee plantations at highest altitude receive less amount of rain precipitation.

Empirical evidence was found between positive attributes of flavor and fragrance of coffee, altitude range and producing regions. So, Montecillos and Opalaca, both at the higher altitude ranges - as have been proven in the correspondence analysis between altitude ranges and coffee producing regions-are, both of them, associated with positive attribute (aroma, body, flavor, acidity and aftertaste).

(3) The crop altitude ranges were found also related to growing regions.

Low altitude was associated to Agalta Tropical, Medium was associated to Azul Meambar and Copan and, finally, high altitude was associated to Opalaca and Montecillos. This result links producing regions by their altitude ranges in meters over sea level, with Opalaca and Montecillos being the higher range mountains and Agalta Tropical lower range mountains.

(4) Empirical evidence was found between agronomic varieties and altitude range. Pacas variety was associated to low altitude, Lempira, Villa Sarchi and IHCAFE-90 to medium altitude and the arabica's varieties Catuai, Caturra to high altitude. Catai and Caturra are pure arabica varieties, in contrast, Lempira and IHCAFE-90 which are hybrid arabica. So, in the medium and low heights, hybrids between arabica and robusta were found: Lempira, IHCAFE-90, Villa Sarchi and Pacas varieties (natural mutations of arabica).

\section{Recommendations}

The first recommendation is an active national coffee policy to develop coffee cultivation, especially at high altitude. The results indicate rich profiles of flavors and fragrances in the Honduran coffee by arabica varieties that can be a strong value in the international markets. The negative flavors as peanuts, grassy and astringent were in relation to the processing and internal trading of coffee, so it was recommended to increase the policy actions aimed at preserving coffee quality. It is necessary to improve coffee technology transference and processing facilities to get a better regulation of marketing relationships between growers and intermediaries.

The second recommendation is about a better resource allocation per region, linking crop altitude ranges to agronomic varieties and to flavors and fragrances. These empirical findings show the marketing potential of using characteristics of coffee that come from specific producing regions.

The third recommendation is to keep the marketing campaign to promote the goodness of the Honduran coffee. The rich profile of flavors and fragrances 
found in the coffee could be very supportive of any international marketing campaign to achieve better pricing strategies. These findings can also be a good opportunity to carry out better business, if private and public leaders can successfully link coffee quality conservation to local growers and flawless traceability from the place of origin to the final destination.

The fourth recommendation is to support more research and development within the coffee industry and with this information to achieve better farm management practices, particularly technical and economic assistance to farmers with differentiated coffee.

\section{Acknowledgments}

Special thanks to the IHCAFE and its coffee quality control lab, the coffee experts Dr. Shawn Steiman, Spencer Turer and Ashley Prentice, among other experts Dr. Abelino Pitty and Dr. Javier Bilbao and all coffee producers who helped in one way or another to this coffee research. The authors would also like to thank the reviewers for their contributions.

\section{References}

[1] Briones, G. T. 1994. Agricultural Sectoral Profile of Honduras. Tegucigalpa: IICA Honduras. (in Spanish)

[2] Arrivillaga, E. D., Robleda, R., and Salomon, L. 1997. Honduras: Toward an Alternative Economic Policy. Tegucigalpa: CEDOH-CH-Honduras. (in Spanish)

[3] De Oliva, A. A. G. 2001. Honduran Rulers, 19th and 20th Centuries. Tegucigalpa: Universitaria UNAH. (in Spanish)

[4] Ortega, P. 1951. The Cultivation of Coffee in Honduras. Tegucigalpa: National Typesetting Company. (in Spanish)

[5] Sivetz, M., and Foote, H. E. 1963. Coffee Processing Technology. Westport, Connecticut: The Avi Publishing Company, Inc..

[6] Jansen, L. 1993. "Coffee and Forms of Production in Honduras.” Central American Journal of Economics 14 (41): 58-96. (in Spanish)

[7] Wagner, R. 2001. History of Coffee from Guatemala. Bogotá: Benjamín Villegas \& Partners. (in Spanish)

[8] International Trade Center (ITC). 2016. The Coffee Exporters' Guide. Genève: INTRACEN-UNCTADCNUCED-WTO.
[9] Rena, A. B., Malavolta, E., Rocha, M., and Yamada, T. 2002. The Coffee Crop: Factors That Affect Productivity. Parasicaba, SP: Brazilian Association for Researching of Phosphorus and Potassium. (in Portuguese)

[10] Sevilla, J. U. 2013. Central American Agricultural Export Analysis: An Economical and Sustainable Evaluation of Coffee Production of Honduras. Ph.D. thesis, King John Charles University. (in Spanish)

[11] Roa, G. M. 1999. Coffee Ecological Benefits. Chinchiná, Colombia: CENICAFE. (in Spanish)

[12] Lopez Camposeco, J. A. 2005. Manual of Wet Coffee. Guatemala City: Guatemala National Coffee Association (Anacafe), USAID and Spanish Cooperation. (in Spanish)

[13] The Inter-American Institute for Cooperation on Agriculture (IICA) and Honduran Coffee Institute (IHCAFE). 2003. Analysis of the Coffee Chain of Honduras. Tegucigalpa: IICA \& the Central Bank of Honduras. (in Spanish)

[14] Deugd, M. 2003. The Coffee Crisis: New Strategies and Opportunities. San José: Ruta-FAO. (in Spanish)

[15] Flores, M., Bratescu, A., Martinez, J. O., Oviedo, J. A., and Acosta, A. 2002. Central America: The Impact of the Fall of the Coffee. México: CEPAL. (in Spanish)

[16] Gressel, C., and Tickel, S. 2002. Mugged: Poverty in Your Coffee Cup. Oxford, UK: Oxfam.

[17] International Coffee Organization (ICO). 2016. Coffee Annual Reports 2000-2016. http://www.ico.org.

[18] Specialty Coffee Association of America (SCAA). 2016. SCAA Protocols: Cupping Specialty Coffee. Santa Ana, California: The Specialty Coffee Association of America (SCAA).

[19] International Coffee Organization (ICO). 2014 Coffee Annual Reports 2000-2014. http://www.ico.org.

[20] Teuber, R. 2008. Geographical Indications and the Value of Reputation-Empirical Evidence for Café of Marcala. Giessen, Germany: Justus Liebig University, Institute for Agricultural Policy and Market Research.

[21] United Nation Development Program (UNDP). 2014. Reports on Human Development: Honduras 2009-2011. Accessed April 4, 2015. http://www.undp.org. (in Spanish)

[22] Central Bank of Honduras (BCH). 2016. Annual Reports 2000-2016. Accessed September 7, 2017. http://www.bch.hn. (in Spanish)

[23] Honduran Coffee Institute (IHCAFE). 2014. Closure Crops Reports (2004-2014). Tegucigalpa: IHCAFE. (in Spanish)

[24] Specialty Coffee Association of America (SCAA). 2014. Specialty Coffee Association of America. Accessed January 25, 2015. http://www.scaa.org.

[25] Alliance for Coffee Excellence (ACE). 2014. The Cup of Excellence Program. Accessed February 20, 2015. 
Findings from Honduras

http://www.cupofexcellence.org.

[26] Yeretzian, C., Glöss, A., Petrozzi, S., D’Ambrosio, L., Knöpfli-Lengweiler, K., Wieland, F., Fridolin, A., and Anliker, R. 2010. "The Smell of Coffee-Analytical Perspective.” New Food Magazine. Issue 4.

[27] Di Donfrancesco, B., Gutierrez Guzman, N., and Chambers, E. 2014. "Comparison of Results from Cupping and Descriptive Sensory Analysis of Colombian Brewed Coffee.” Journal of Sensory Studies 29 (4): 301-11.

[28] Chin, S. T., Graham, T. E., and Marriott, P. J. 2011. "Identification of Potent Odourants in Wine and Brewed Coffee Using Gas Chromatography-Olfactometry and Comprehensive Two-Dimensional Gas Chromatography.” J. Chromatogr. A. 1218 (42): 7487-98.

[29] Krejcie, R. V., and Morgan, D. W. 1970. "Determining Sample Size for Research Activities.” Educational and Psychological Measurement 30 (3): 607-10.

[30] Hernández, R., Fernández, C., and Bautista, P. 2008. Research Methodology. México, DF: Mcgraw Hill. (in Spanish)

[31] Lara-Estrada, L. 2007. "Effects of Altitude, Shade, Production and Fertilization on the Quality of the Coffee (Coffee arabica L. var. caturra) Produced in Agroforestry Systems of the Central Coffee Zone of Nicaragua." Presented at the Second International Symposium on
Multi-strata Agroforestry Systems with Perennial Crops, IUFRO, CIRAD, CATIE, Costa Rica. (in Spanish)

[32] Di Rienzo, J. A., Casanoves, F., González, L. A., and Balzarini, M. 2008. Statistics for the Agricultural Sciences. 4th ed.. Córdoba, Argentina: Editorial Brujas. (in Spanish)

[33] Balzarini, M. G., González, L., Tablada, M., Casanoves, F., Di Rienzo, J. A., and Robledo, C. W. 2008. User Manual. Córdoba, Argentina: Editorial Brujas.

[34] Jiménez, E., and Manzano, J. 2005. Applied Multivariate Analysis. Madrid: Thomson. (in Spanish)

[35] Rencher, A. C. 2002. Methods of Multivariate Analysis. 2nd ed.. New York: John Wiley \& Sons, Inc.

[36] Andino, J. 2004. "Evaluating International Agricultural Trade in Central America: Three Analyses.” Ph.D. thesis, Louisiana State University.

[37] Damiani, O. 2005. In the Face of Adversity and Change, Successful Strategies of Small Coffee Producers in Central America. San José: Ruta-FAO. (in Spanish)

[38] Sanetra, C., and Goslar, F. 2007. The Global Quality Challenge-A National Quality Infrastructure. Berlín: PTB-OEA. (in Spanish)

[39] Fitter, R., and Kaplinsky, R. 2001. Who Gains from Product Rents as the Market Become More Differentiated? A Value Chain Analysis. Sussex: IDS-University of Sussex. 